\title{
Respiratory Aspects of Spinal Cord Injury Management
}

\author{
R. Edward Carter, M.D. \\ Vice-President, Medical Affairs, The Institute for Rehabilitation and Research, \\ 1333 Moursund Avenue, Houston, TX 77030. Professor, Clinical Rehabilitation, \\ Baylor College of Medicine, One Baylor Plaza, Houston, TX 77030, U.S.A.
}

Cardiopulmonary problems now outrank problems of the urinary system as the most common problems involved in the death of patients with spinal cord injury, both during the acute phase and also during later years, especially in quadriplegics.

The National Spinal Cord Injury Data Base, compiling data from 13 Regional Spinal Cord Injury Centers in the United States, reported occurrences of medical complications during the initial medical/rehabilitation period. The latest report covered 9647 spinal cord injury cases admitted to the spinal cord injury systems within 24 hours of injury. Diseases of the respiratory system are the leading cause of death, with most respiratory deaths attributed to pneumonia $\left(78 \cdot 5^{\circ}{ }_{0}\right)$.

A series of 2619 patients admitted to The Institute for Rehabilitation and Research (TIRR) from 1959 through 1985 are separated by functional motor levels, as indicated in Table I. These functional motor levels represent the most inferior muscle grading fair to above at the time of admission to the Spinal Cord Injury Center. Thus, one can see, $4 \cdot 1^{\circ}{ }_{0}$ fall into the apneic group, $46 \cdot 5^{\circ}$ o into the usual quadriplegic group, and $17 \cdot 3^{\circ}$ o into the high paraplegic category. Thus, a total of $67.9^{\circ}$ o of all spinal cord injury may be expected to have a marked predisposition for respiratory problems.

\section{Medical management of the usual quadriplegic (C4 through C8)}

Initial medical management must start with an adequate history which includes those variables that may prolong or intensify medical therapy. The history should include the age of the patient, a past history of smoking, pulmonary disease, and/or pulmonary allergy. Associated injuries or other complications of spinal injury must also be noted. The physical and neurological examination should be thorough, particularly with regards to the respiratory system where attention should be paid to use of accessory musculature, the symmetry of 
Table I The Institute for Rehabilitation and Research Texas/South Central Regional Spinal Cord Injury System Survey of Spinal Cord Injury Functional Motor Level Distribution: 1959-1985

\begin{tabular}{ccc}
\hline Level of injury & Number of Patients & ${ }^{\circ}{ }_{0}$ of Total \\
\hline C-1/C-3 & 108 & $4 \cdot 1 \%$ \\
C-4 & 221 & $8 \cdot 4^{\circ}$ \\
C-5 & 386 & $14 \cdot 7^{\circ}$ \\
C-6 & 379 & $14 \cdot 5^{\circ}$ \\
C-7 & 186 & $7 \cdot 1 \%$ \\
C-8 & 46 & $1 \cdot 8 \%$ \\
Quadriplegia sub-total & 1326 & $50 \cdot 6^{\circ} \%$ \\
T-1/T-3 & 86 & $3 \cdot 3 \%$ \\
T-4/T-7 & 366 & $14 \cdot 0^{\circ}$ \\
T-8/T-11 & 312 & $12 \cdot 0^{\circ}$ \\
T-12/L-2 & 452 & $17 \cdot 2 \%$ \\
L-3/L-5 & 76 & $2 \cdot 9 \%$ \\
Paraplegia sub-total & 1293 & $49 \cdot 4 \%$ \\
Grand total-27 years & 2619 & $100 \cdot 0^{\circ} \%$ \\
\hline
\end{tabular}

Motor level from first admission to spinal cord rehabilitation program

breathing, pattern of breathing, etc. In addition to the usual laboratory determinations, (bloodcount, electrolytes, etc.), arterial blood gases should be determined as well as vital capacity, chest x-ray, and flat plate of the abdomen. Initial respiratory therapy starts with a face mask for extra humidity and intermittent positive pressure breathing four times daily with the addition of bronchodilators and mucolytics to the nebulizer. IPPB is followed by chest clapping and assistive coughing and/or suctioning. Oxygen is added to the face mask depending on the determination of the arterial oxygen concentration. Should there be some question of the chest symmetry during respiration and fluroscopy is not feasible, then double exposure $x$-ray films, in deep inspiration and in deep expiration, should be taken to rule out a paralytic diaphragm.

A major goal is avoidance of hypoxia. The problem is an unopposed vagus nerve which tends to bradycardia and may tend to have transient episodes of cardiac block, particularly on stimulation of the vagus. The patient should be placed on a cardiac monitor for the first 10 to 14 days with a vital capacity checked each nursing shift for the first 10 days and periodic determinations of arterial blood gases.

The average vital capacity in the usual quadriplegic patient, with accessory respiratory muscles and diaphragm but without intercostal muscles and abdominal muscles, should be about $2200 \mathrm{cc}$ to $2500 \mathrm{cc}$. However, in a series of 50 quadriplegics followed from the first 24 to 48 hours at (TIRR), the average initial vital capacity was $1250 \mathrm{cc}$. If this vital capacity is below $1000 \mathrm{cc}$ to 1100 $\mathrm{cc}$ in an adult, then further pathology such as unilateral diaphragmatic paralysis should be suspected.

The major problem time is from the 3rd to the 5 th day post-trauma when pooling of the secretions may lead to atelectasis and then to pneumonia. Patients should be followed with serial vital capacities until they average $1400 \mathrm{cc}$ to 1500 cc. At that time incentive respirometry should be added two to three times daily. As soon as the patient is cleared from the standpoint of neck stability, isometric exercises to the neck muscles and strengthening to accessory muscles 
of respiration are begun. This latter therapy becomes far more important the higher the level of the spinal cord lesion to initiate early post-trauma.

A major complication is atelectasis which occasionally occurs coincidentally with diaphragmatic fatigue. A relatively recent study by Gross, et al. (1980), concluded that quadriplegics are predisposed to the development of inspiratory muscle fatigue due to reduced muscle strength and reduction in endurance and secondly, in these patients, inspiratory muscle training increases both strength and endurance and protects against fatigue. Should atelectasis and/or pneumonitis occur, the patient should be placed on intermittent positive pressure breathing every 4 hours followed by postural drainage and chest clapping to drain the affected areas. This is followed with assisted coughing and/or suctioning. Antibiotics should be utilised according to the sensitivities derived from sputum or tracheal cultures. Should these procedures fail to mobilise or clear atelectatic areas, then bronchoscopy should be employed. Chest $\mathrm{x}$-rays should be performed every 48 hours because of the possibility of 'migrating' atelectasis which mandates a change in the patient's postural drainage and chest clapping. In the event that the patient is unable to maintain adequate ventilation, then tracheotomy and mechanical ventilation is necessary. The two major goals of mechanical ventilation are: (1) to maintain an alveolar ventilation appropriate for the patient's metabolic requirements and (2) to correct arterial hypoxemia and maximise oxygen transport. Tracheotomy tubes should be of soft plastic material and care should be taken to allow at least a minimal air leak around the tracheotomy tube and/or cuff. Period fiberoptic bronchoscopy should be performed in any patient who is on a prolonged tracheotomy or endotracheal tube.

Weaning of the patient from mechanical ventilation is not infrequently done much too rapidly and outstrips the endurance of the diaphragm resulting in a fatigue syndrome. Our method is to continue the patients on mechanical ventilation and IPPB until the vital capacity reaches $1200 \mathrm{cc}$ to $1400 \mathrm{cc}$ in the supine position. The patient is initially weaned off positive and expiratory pressure, if this has been utilised, and then weaned from additional increments of oxygen down to room air. If this is tolerated, the patient is then allowed to go on a Ttube for 1 hour, three to four times daily. This time is gradually increased to his tolerance with periodic checking of arterial blood gases at the end of the T-tube time. Incentive respirometry is then added several times daily and as the patient reaches a supine vital capacity of $1500 \mathrm{cc}$, he is then usually able to tolerate sitting without dropping the vital capacity to dangerously low levels. The possible use of Aminophylline to improve the contractural force of the human diaphragm and to reverse fatigue needs to be studied in the presence of hypoxemia and in the ventilatory dependent quadriplegia.

\section{Prognosis or results}

C3 quadriplegics-approximately 30\% of this apneic group are due to ascending neurologic lesions that after a period of usually 60 to 75 days prove to be reversible to the point of recovering some volitional breathing (Table II). Most gain enough vital capacity to be weaned from respiratory equipment. The average improvement in their vital capacity is $1080 \mathrm{cc}$.

C4 quadriplegics-previous studies on complete motor C4 quadriplegics had 
Table II The Institute for Rehabilitation and Research Respiratory Study 1959-1978

\begin{tabular}{lccccc}
\hline \multicolumn{1}{c}{ Classification } & $\begin{array}{c}\text { Number } \\
\text { of } \\
\text { patients }\end{array}$ & $\begin{array}{c}\text { Average } \\
\text { initial } \\
\text { V.C. (cc) }\end{array}$ & $\begin{array}{c}\text { Average } \\
\text { final } \\
\text { V.C. (cc) }\end{array}$ & $\begin{array}{c}\text { Average } \\
\text { gain in } \\
\text { V.C. (cc) }\end{array}$ & $\begin{array}{c}\text { Average duration } \\
\text { initial to final } \\
\text { V.C. (days) }\end{array}$ \\
\hline $\begin{array}{l}\text { C-3 } \\
\text { With return) }\end{array}$ & 6 & 100 & 1180 & 1080 & - \\
$\begin{array}{l}\text { C-4 } \\
\text { (Initial V.C. }<1000 \mathrm{cc} \text { ) }\end{array}$ & 26 & 670 & 1837 & 1167 & 75.5 \\
$\begin{array}{l}\text { C-4 } \\
\text { (Initial V.C. > 1000 cc) }\end{array}$ & 19 & 1550 & 2672 & 1122 & 57 \\
$\begin{array}{l}\text { Unilateral diaphragmatic } \\
\text { paralysis }\end{array}$ & & & & & \\
$\quad \begin{array}{l}\text { A. Transient } \\
\text { B. Permanent }\end{array}$ & 16 & 1019 & 2151 & 1132 & 76 \\
\hline
\end{tabular}

grouped these into those who presented with initial vital capacity below $1000 \mathrm{cc}$ and a second group who presented with their initial vital capacity above $1000 \mathrm{cc}$ (Carter et al., 1979-80) (Table II). In the first group, the average improvement in vital capacity was $1167 \mathrm{cc}$ while the second group improved by $1122 \mathrm{cc}$. Similar figures of improvement in vital capacities are shown for those patients presenting with both permanent and with transient unilateral diaphragmatic paralysis (Carter et al., 1980).

\section{Mortality}

The mortality figures for spinal cord injury are continually decreasing. The greatest increase in mortality occurs in the first 6 months to 1 year post-trauma. After the first 1 to 2 years, the mortality curve parallels that of normal individuals. Even in high quadriplegics, those discharged from spinal cord injury centres requiring mechanical ventilatory support, the mortality rates continue to markedly decrease. Comparison of three consecutive year periods, 1963 to 1969,1970 to 1977 , and 1978 to 1985 , have shown an approximate $50 \%$ decrease in mortality of $\mathrm{C} 4$ and higher quadriplegics from each period to the next. A multi-spinal cord injury centre survey (Whiteneck et al., 1985) of high quadriplegics completed in November, 1985 and surveying 216 quadriplegics, defined as level of $\mathrm{C} 4$ complete or above, revealed survivals substantially different between respirator dependent and respirator independent cases. At 1, 3, 5, 7, and 9 years injury, the survival percentages of the respirator independent group were $93 \%, 83 \%, 79 \%, 77 \%$, and $73 \%$ respectively. For the respirator dependent group the survival rates were $86 \%, 70 \%, 63^{\circ}, 59^{\circ} \%$ and $63 \%$ respectively.

Additional research efforts should be directed towards improving the safety of patients on long-term tracheotomies and the comparison of the types of tracheotomy tubes for prevention of endotracheal damage. Better understanding of the mechanics of atelectasis and chronic endobronchial infection is also necessary for the percentage of respiratory complications in spinal cord injuries remains much too high. Certainly there are few, if any, areas elsewhere in the field of medicine that require treatment prior to the onset of pathology as does the respiratory muscle weakness in spinal cord injury. 


\section{Acknowledgement}

This work was in part supported by the National Institute of Handicapped Research Grant \#133NH50014.

\section{References}

CARTER RE 1979-1980 Experiences with high tetraplegics. Paraplegia 17: 140-146.

CARTER RE 1980 Unilateral diaphragmatic paralysis in spinal cord injury patients. Paraplegia 18: 267-273.

Gross D, LADD HW, RILEY EJ, et al. 1980 The effect of training on strength and endurance of the diaphragm in quadriplegia. The American fournal of Medicine 68: 27-35.

Whiteneck GG, Carter RE, Charlifue SW, et al. 1985 A collaborative study of high quadriplegia. Grant Report, U.S. Department of Education, Rehabilitation Research and Demonstrations-Field Initiated Research. Contact: Gayle Whiteneck, Ph.D. (Craig Hospital, Englewood, Colorado). 